Document downloaded from:

http://hdl.handle.net/10251/66351

This paper must be cited as:

Cordero Barbero, A.; Franqués García, AM.; Torregrosa Sánchez, JR. (2015). Multidimensional Homeier's generalized class and its application to planar 1D Bratu problem. Journal of the Spanish Society of Applied Mathematics. 70(1):1-10. doi:10.1007/s40324-015-0037-x.

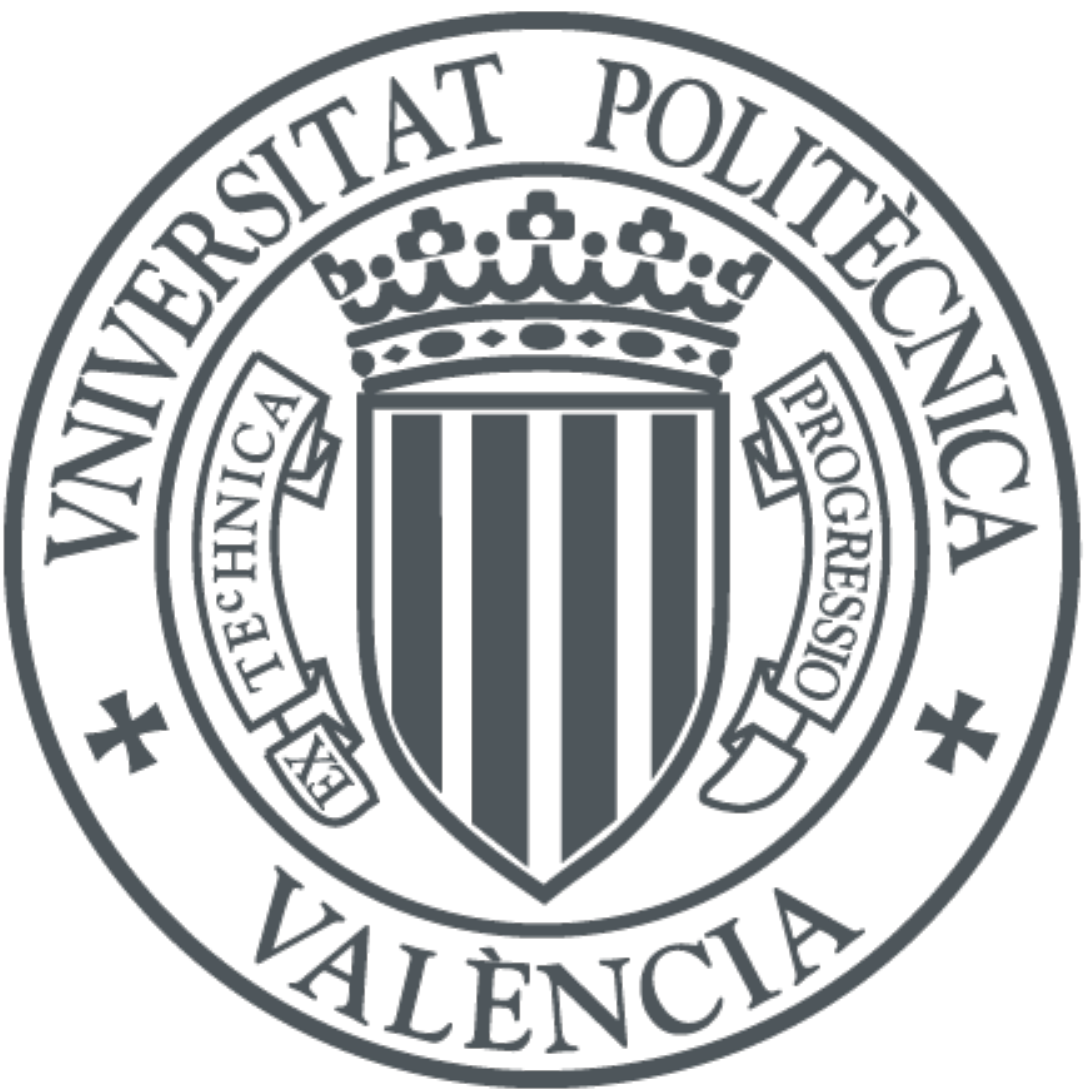

The final publication is available at

http://dx.doi.org/10.1007/s40324-015-0037-x

Copyright Springer

Additional Information 


\title{
Multidimensional Homeier's generalized class and its application to planar 1D Bratu problem
}

\author{
Alicia Cordero · Antonio Franques · Juan R. Torregrosa
}

Received: date/ Accepted: date

\begin{abstract}
In this paper, a parametric family of iterative methods for solving nonlinear systems, including Homeier's scheme is presented, proving its third-order of convergence. The numerical section is devoted to obtain an estimation of the solution of the classical Bratu problem by transforming it in a nonlinear system by using finite differences, and solving it with different elements of the iterative family.
\end{abstract}

Keywords Nonlinear system · iterative method · order of convergence $\cdot$ Bratu's problem

Mathematics Subject Classification (2000) $65 \mathrm{H} 10 \cdot 49 \mathrm{M} 15$

\section{Introduction}

Problems in science and engineering usually involve nonlinear equations or systems $F(x)=0$, where $F: D \subseteq \mathbb{R}^{n} \rightarrow$ $\mathbb{R}^{n}, n \geq 1$. The exact solutions $\alpha \in \mathbb{R}^{n}$ of these kind of problems are difficult to find and, frequently, we must use iterative methods in order to estimate these solutions.

A way to design iterative methods for solving nonlinear systems is to adapt the methods of the scalar case, but these schemes not always can be extended to multidimensional case, at least, in a direct form. There exist some methods in the scalar case which are directly extended to systems of nonlinear equations, others have been specially designed for systems.

In the last years, different procedures have been used in the development of iterative methods for nonlinear systems, see for example the papers of Sharma et al. [1], [2], the results presented by Awawdeh in [3] and by Kanwar et al. in [4], the schemes published by Andreu et al. in [5], applied by the authors for solving the problem of preliminary orbit determination of artificial satellites, and the work of Abad et al. [6], where the authors apply the designed methods on the Global Positioning System.

The proliferation of iterative methods for solving nonlinear equations has been spectacular in the last years (in [7] we can see a good review). Some of these methods can be transferred easily to the context of nonlinear systems, but others, at least apparently, cannot be extended to multidimensional case. One of the schemes of the first type is Homeier's method, described in [8] and whose iterative expression for nonlinear systems is

$$
\begin{aligned}
y^{(k)} & =x^{(k)}-F^{\prime}\left(x^{(k)}\right)^{-1} F\left(x^{(k)}\right), \\
x^{(k+1)} & =x^{(k)}-\left[F^{\prime}\left(y^{(k)}\right)^{-1} F\left(x^{(k)}\right)+F^{\prime}\left(x^{(k)}\right)^{-1} F\left(x^{(k)}\right)\right],
\end{aligned}
$$

In this paper, we present a parametric family of iterative methods for solving nonlinear systems as a generalization of the previous scheme, whose iterative expression is:

$$
\begin{aligned}
y^{(k)} & =x^{(k)}-\varphi F^{\prime}\left(x^{(k)}\right)^{-1} F\left(x^{(k)}\right), \\
x^{(k+1)} & =x^{(k)}-\left[\gamma F^{\prime}\left(y^{(k)}\right)^{-1} F\left(x^{(k)}\right)+\delta F^{\prime}\left(x^{(k)}\right)^{-1} F\left(x^{(k)}\right)\right],
\end{aligned}
$$

This research was supported by Ministerio de Economía y Competitividad MTM2014-52016-C02-02

Alicia Cordero · Antonio Franques · Juan R. Torregrosa

Instituto Universitario de Matematica Multidisciplinar, Universitat Politècnica de València, Camino de Vera, s/n, 46022 Valencia, Spain

E-mail: acordero@mat.upv.es, anfragar@teleco.upv.es,jrtorre@mat.upv.es 
where $\varphi, \gamma$ and $\delta$ are real parameters. As we will prove in Section 2, the methods of family (2) have order of convergence three from different relations between the parameters.

We recall that a sequence $\left\{x^{(k)}\right\}_{k \geq 0}$ converges to $\alpha$ with order of convergence $p$ if there exist a positive constant $C$ such that

$$
\lim _{k \rightarrow \infty} \frac{\left\|x^{(k+1)}-\alpha\right\|}{\left\|x^{(k)}-\alpha\right\|^{p}}=C .
$$

The rest of the paper is organized as follows: the order of convergence of family (2) is analyzed in Section 2. In Section 3, the planar 1D Bratu problem is studied, obtaining numerical results from different elements of the family and comparing them with some known methods. Finally, some remarks and conclusions are presented.

\section{Convergence analysis}

The next result describes the conditions under which family (2) has order of convergence three. In the proof we use the notations and tools presented in [9].

Let $F: D \subseteq \mathbb{R}^{n} \longrightarrow \mathbb{R}^{n}$ be sufficiently differentiable in $D$. The $q$ th derivative of $F$ at $u \in \mathbb{R}^{n}, q \geq 1$, is the $q$-linear function $F^{(q)}(u): \mathbb{R}^{n} \times \cdots \times \mathbb{R}^{n} \longrightarrow \mathbb{R}^{n}$ such that $F^{(q)}(u)\left(v_{1}, \ldots, v_{q}\right) \in \mathbb{R}^{n}$. It is easy to observe that

1. $F^{(q)}(u)\left(v_{1}, \ldots, v_{q-1}, \cdot\right) \in \mathscr{L}\left(\mathbb{R}^{n}\right)$

2. $F^{(q)}(u)\left(v_{\sigma(1)}, \ldots, v_{\sigma(q)}\right)=F^{(q)}(u)\left(v_{1}, \ldots, v_{q}\right)$, for all permutation $\sigma$ of $\{1,2, \ldots, q\}$.

From the above properties we can use the following notation:

(a) $F^{(q)}(u)\left(v_{1}, \ldots, v_{q}\right)=F^{(q)}(u) v_{1} \ldots v_{q}$

(b) $F^{(q)}(u) v^{q-1} F^{(p)} v^{p}=F^{(q)}(u) F^{(p)}(u) v^{q+p-1}$

On the other hand, for $\alpha+h \in \mathbb{R}^{n}$ lying in a neighborhood of a solution $\alpha$ of $F(x)=0$, we can apply Taylor's expansion and assuming that the jacobian matrix $F^{\prime}(\alpha)$ is nonsingular, we have

$$
F(\alpha+h)=F^{\prime}(\alpha)\left[h+\sum_{q=2}^{p-1} C_{q} h^{q}\right]+O\left(h^{p}\right),
$$

where $C_{q}=(1 / q !)\left[F^{\prime}(\alpha)\right]^{-1} F^{(q)}(\alpha), q \geq 2$. We observe that $C_{q} h^{q} \in \mathbb{R}^{n}$ since $F^{(q)}(\alpha) \in \mathscr{L}\left(\mathbb{R}^{n} \times \cdots \times \mathbb{R}^{n}, \mathbb{R}^{n}\right)$ and $\left[F^{\prime}(\alpha)\right]^{-1} \in \mathscr{L}\left(\mathbb{R}^{n}\right)$.

In addition, we can express $F^{\prime}$ as

$$
F^{\prime}(\alpha+h)=F^{\prime}(\alpha)\left[I+\sum_{q=2}^{p-1} q C_{q} h^{q-1}\right]+O\left(h^{p}\right)
$$

where $I$ is the identity matrix. Therefore, $q C_{q} h^{q-1} \in \mathscr{L}\left(\mathbb{R}^{n}\right)$. From (4), we obtain

$$
\left[F^{\prime}(\alpha+h)\right]^{-1}=\left[I-X_{2} h+X_{3} h^{2}-X_{4} h^{4}+\cdots\right]\left[F^{\prime}(\alpha)\right]^{-1}+O\left(h^{p}\right),
$$

where

$$
\begin{aligned}
& X_{2}=2 C_{2} \\
& X_{3}=4 C_{2}^{2}-3 C_{3}, \\
& X_{4}=8 C_{2}^{3}-6 C_{2} C_{3}-6 C_{3} C_{2}+4 C_{4}
\end{aligned}
$$

We denote $e^{(k)}=x^{(k)}-\alpha$ the error in the $k$ th iteration in the scalar and multidimensional case, respectively. The equation

$$
e^{(k+1)}=L e^{(k) p}+O\left(e^{(k)^{p+1}}\right),
$$

where $L$ is a $p$-linear function $L \in \mathscr{L}\left(\mathbb{R}^{n} \times \cdots \times \mathbb{R}^{n}, \mathbb{R}^{n}\right)$, is called the error equations and $p$ is the order of convergence. Observe that $e^{(k)^{p}}$ is $\left(e^{(k)}, e^{(k)}, \cdots, e^{(k)}\right)$. 
Theorem 1 Let $\alpha \in D$ be a zero of a sufficiently differentiable function $F: D \subseteq \mathbb{R}^{n} \rightarrow \mathbb{R}^{n}$ in a convex set $D$ with non-singular Jacobian matrix $F^{\prime}(x)$ in $\alpha$. If $\varphi=\frac{1}{2 \gamma}$ and $\delta=1-\gamma$, then the schemes of family (2) provides third-order of convergence, whose error equation is given by

$$
e^{(k+1)}=\left[\left(-3+8 \gamma-4 \gamma^{2}\right) C_{2}^{2}+\left(\frac{11}{4}-6 \gamma+3 \gamma^{2}\right) C_{3}\right] e^{(k)^{3}}+O\left(e^{(k)^{4}}\right),
$$

where $C_{k}=\frac{1}{k !}\left[F^{\prime}(\alpha)\right]^{-1} F^{(k)}(\alpha), k=2,3, \ldots$ and $e^{(k)}=x^{(k)}-\alpha$

Proof: If we use Taylor expansion of $F\left(x^{(k)}\right)$ and $F^{\prime}\left(x^{(k)}\right)$ around $\alpha$, we obtain

$$
F\left(x^{(k)}\right)=F^{\prime}(\alpha)\left\{e^{(k)}+C_{2} e^{(k)^{2}}+C_{3} e^{(k)^{3}}\right\}+O\left(e^{(k)^{4}}\right)
$$

and

$$
F^{\prime}\left(x^{(k)}\right)=F^{\prime}(\alpha)\left\{I+2 C_{2} e^{(k)}+3 C_{3} e^{(k)^{2}}\right\}+O\left(e^{(k)^{3}}\right)
$$

From (6), we calculate the expression of the inverse

$$
\left[F^{\prime}\left(x^{(k)}\right)\right]^{-1}=\left\{I+X_{2} e^{(k)}+X_{3} e^{(k)^{2}}\right\}\left[F^{\prime}(\alpha)\right]^{-1}+O\left(e^{(k)^{3}}\right),
$$

where $X_{2}=-2 C_{2}, X_{3}=4 C_{2}^{2}-3 C_{3}$ and $I$ denotes the identity matrix of size $n \times n$.

These values have been obtained by imposing the conditions

$$
\left[F^{\prime}\left(x^{(k)}\right)\right]^{-1} F^{\prime}\left(x^{(k)}\right)=F^{\prime}\left(x^{(k)}\right)\left[F^{\prime}\left(x^{(k)}\right)\right]^{-1}=I .
$$

Then, the error expression in the first step of the method is

$$
e_{y}^{(k)}=y^{(k)}-\alpha=(1-\varphi) e^{(k)}+\varphi C_{2} e^{(k)^{2}}+O\left(e^{(k)^{3}}\right) .
$$

Furthermore, we know that

$$
F^{\prime}\left(y^{(k)}\right)=F^{\prime}(\alpha)\left\{I+2 C_{2} e_{y}^{(k)}+3 C_{3} e_{y}^{(k)^{2}}\right\}+O\left(e_{y}^{(k)^{3}}\right)
$$

In a similar way as in (7),

$$
\left[F^{\prime}\left(y^{(k)}\right)\right]^{-1}=\left\{I+Y_{2} e_{y}^{(k)}+Y_{3} e_{y}^{(k)^{2}}\right\}\left[F^{\prime}(\alpha)\right]^{-1}+O\left(e_{y}^{(k)^{3}}\right),
$$

where $Y_{2}=-2 C_{2}$ and $Y_{3}=4 C_{2}^{2}-3 C_{3}$.

And, if we replace in (9) the powers of $e_{y}^{(k)}=y^{(k)}-\alpha$, we obtain after some algebraic operations

$$
\begin{aligned}
{\left[F^{\prime}\left(y^{(k)}\right)\right]^{-1}=} & \left\{I-2(1-\varphi) C_{2} e^{(k)}+\left[\left(4-10 \varphi+4 \varphi^{2}\right) C_{2}^{2}+\left(-3+6 \varphi+3 \varphi^{2}\right) C_{3}\right] e^{(k)^{2}}\right\}\left[F^{\prime}(\alpha)\right]^{-1} \\
& +O\left(e^{(k)^{3}}\right) .
\end{aligned}
$$

Finally, the error equation is expressed as

$$
\begin{aligned}
e^{(k+1)}= & (1-\gamma-\delta) e^{(k)}+(\gamma+\delta-2 \varphi \gamma) C_{2} e^{(k)^{2}} \\
& +\left[\left(8 \varphi \gamma-2 \gamma-2 \delta-4 \varphi^{2} \gamma\right) C_{2}^{2}+\left(2 \gamma+2 \delta-6 \varphi \gamma+3 \varphi^{2} \gamma\right) C_{3}\right] e^{(k)^{3}}+O\left(e^{(k)^{4}}\right)
\end{aligned}
$$

If we force that the terms corresponding to $e^{(k)}$ and $e^{(k)^{2}}$ are eliminated in (11), we have the following system:

$$
\left.\begin{array}{l}
1-\gamma-\delta=0 \\
\gamma+\delta-2 \varphi \gamma=0
\end{array}\right\}
$$

whose solution is $\varphi=\frac{1}{2 \gamma}$ and $\delta=1-\gamma$. This solution is unique, although it depends on a free parameter, $\gamma$. If we replace $\varphi$ and $\delta$ in (2) we obtain a third-order family of iterative methods, which error equation can be expressed as:

$$
e^{(k+1)}=\left[\left(-3+8 \gamma-4 \gamma^{2}\right) C_{2}^{2}+\left(\frac{11}{4}-6 \gamma+3 \gamma^{2}\right) C_{3}\right] e^{(k)^{3}}+O\left(e^{(k)^{4}}\right)
$$


and the proof is finished.

We denote the members of the obtained family

$$
\begin{aligned}
y^{(k)} & =x^{(k)}-\frac{1}{2 \gamma} F^{\prime}\left(x^{(k)}\right)^{-1} F\left(x^{(k)}\right), \\
x^{(k+1)} & =x^{(k)}-\left[\gamma F^{\prime}\left(y^{(k)}\right)^{-1} F\left(x^{(k)}\right)+(1-\gamma) F^{\prime}\left(x^{(k)}\right)^{-1} F\left(x^{(k)}\right)\right],
\end{aligned}
$$

by $H G(\gamma)$, some of them will be used in the numerical section. On the other hand, regarding error equation (11) some interesting values of parameter $\gamma$ are the roots of $-3+8 \gamma-4 \gamma^{2}=0$, that is, $\gamma=1 / 2$ and $\gamma=3 / 2$, and the roots of $\frac{11}{4}-6 \gamma+3 \gamma^{2}=0, \gamma=(6-\sqrt{3}) / 6$ and $\gamma=(6+\sqrt{3}) / 6$. These values reduce the asymptotical error.

\section{Numerical Results}

In this section, we present the planar 1D Bratu problem [10] and we solve the nonlinear system obtained by applying symmetric finite differences to it, by using iterative methods of class (12).

\subsection{The planar 1D Bratu problem}

We consider the classical Bratu problem which is an elliptic nonlinear partial differential equation with homogeneous Dirichlet conditions on the boundary. The problem is given by

$$
\begin{aligned}
\Delta u+C e^{u}=0 & \text { in } \Omega, \\
u=0 & \text { on } \delta \Omega,
\end{aligned}
$$

where $C>0$ and $\Omega$ is a bounded domain with boundary $\delta \Omega$. The problem is a nonlinear eigenvalue problem that is commonly used as a test problem for many numerical methods. In the planar 1D case, the problem reduces to the expression

$$
\frac{d^{2} u}{d x^{2}}+C e^{u}=0, \quad 0 \leq x \leq 1,
$$

whose boundary conditions are

$$
u(0)=u(1)=0 .
$$

The Bratu problem appears in a large variety of application areas such as the fuel ignition model of thermal combustion, radiative heat transfer, thermal reaction, the Chandrasekhar model of the expansion of the universe, chemical reactor theory and nanotechnology [11-14]. In [12] a summary of the history of the problem is given. The Bratu problem contains parameter $C$ and it has two solutions for $C<C_{c}$ leading to two branches, called lower and upper branches. There is only one solution for $C=C_{c}$ and no solution for $C>C_{c}$, where $C_{c}=3.513830719$ is a critical turning point, which makes the linear part of (17) tangent to $\cosh (\alpha)$.

The exact solution of the planar 1D Bratu problem described in (14) and (15) is given by:

$$
u(x)=2 \ln \left[\frac{\cosh (\alpha)}{\cosh (\alpha(1-2 x))}\right],
$$

where $\alpha$ satisfies the transcendental equation

$$
\cosh (\alpha)=\frac{4}{\sqrt{2 C}} \alpha .
$$

The solution of this equation may be performed by using any iterative method for solving nonlinear equation, for example Newton's scheme, which results can be observed in Table 1, for three values of $C$, such as $C=0.1$, $C=1.0$ and $C=3.0$. Let us notice that depending on the initial estimation of the iterative method, either the value corresponding to the lower or upper branch is obtained. 


\begin{tabular}{|c|c|c|}
\cline { 2 - 3 } \multicolumn{1}{c|}{} & \multicolumn{2}{c|}{ Value of $\alpha$} \\
\hline$C$ & Lower branch & Upper branch \\
\hline 0.1 & 0.1125 & 4.3554 \\
\hline 1.0 & 0.3793 & 2.7347 \\
\hline 3.0 & 0.8434 & 1.6441 \\
\hline
\end{tabular}

Table 1: Both solutions to the equation presented in (17), for different values of $C$.

3.2 The finite difference solution and numerical study of the 1D Bratu problem

The solution of Bratu problem using the finite difference method involves discretizing the differential equation (14) and including the boundary conditions (15). The method transforms the problem into a system of simultaneous nonlinear equations which are then solved using different iterative schemes: Newton's method $(N)$, Traub's method (T) (see [15]) and some elements of family $H G(\gamma)$. Using an uniform grid with step $h=1 /(n+1)$ for an integer $n$, the range $0 \leq x \leq 1$ is discretized as $x_{j}=0+j h$, with $j=0,1, \ldots, n+1$. Using a symmetric standard finitedifference (SFD) scheme, the discrete version of the planar Bratu problem will be

$$
\frac{u_{i+1}-2 u_{i}+u_{i-1}}{h s}+C e^{u_{i}}=0, \quad i=1,2 \ldots, n,
$$

where $h s=h^{2}$, with $u_{0}=u_{n+1}=0$.

On the other hand, a Mickens nonstandard finite difference (NSFD) scheme can be also used [16], which yields

$$
\frac{u_{i+1}-2 u_{i}+u_{i-1}}{h s}+C e^{u_{i}}=0, \quad i=1,2, \ldots, n,
$$

where $h s=2 \ln [\cosh (h)]$. In the limit as $h$ tends to zero, the NSFD reduces to the SFD.

Previous results given by Buckmire in [16] show that the error due to the finite difference decreases proportionally to $h^{2}$. Also the errors of the NSFD are consistently smaller than those of the SFD, as it can be seen in Tables 2 and 3.

For treating the resulting nonlinear system, it is well-known that the solution is much more reliable and obtained much more quickly if the initial guess is near to the solution. Thus, for iteratively solving the resulting nonlinear system, a simple sinusoidal starting function (similar to that used by Boyd [17]) having appropriate amplitude $a$ (which satisfies the boundary conditions) is used. In particular, for the lower branch, $a$ should be taken as $a<u_{c}$, while the upper branch requires $a>u_{c}$ (otherwise, it could happen that due to a wrong initial guess, our method would converge to the opposite solution), where $u_{c}$ is the unique solution of (16) when $C=C_{c}$. We have decided to choose $a=1$ and $a=8$ respectively, as it was suggested in [18], being the sinusoidal starting function

$$
s(x)=a \sin (\pi x) .
$$

We applied the three methods $N, T$ and $H G(\gamma)$ for the three values of $C$ that were also used in Table 1, taking $n=20$ and a stopping criterium $\left\|x^{(k+1)}-x^{(k)}\right\|+\| F\left(x^{(k)} \|<10^{-10}\right.$ for all cases (decreasing the tolerance would not increase the accuracy of the solution, since the bottleneck would still be on the discretization process). A comparison between the greatest error (maximum difference between the exact solution obtained from (16) and the computed by the iterative method) for each value of $C$ as well as for lower/upper branch, is listed in Tables 2 and 3, for SFD and NSFD, respectively. The computed solution obtained by the three methods only differ after the sixteenth decimal, hence the results showed in these tables are the same for all of them. As a matter of fact, in the specific case of $H G(\gamma)$, four members of the family are tested, $\gamma=1 / 2, \gamma=3 / 2, \gamma=(6-\sqrt{3}) / 6$ and $\gamma=(6+\sqrt{3}) / 6$; those corresponding to the values of the parameter that reduce the asymptotical error. The same computed solution has been obtained for the four of them, therefore the results shown in Tables 2 and 3 are identical for the chosen values of the parameter $\gamma$.

\begin{tabular}{|c|c|c|}
\cline { 2 - 3 } \multicolumn{1}{c|}{} & \multicolumn{2}{c|}{ Greatest error } \\
\hline$C$ & Lower branch & Upper branch \\
\hline 0.1 & $2.8802 \mathrm{e}-6$ & 0.0185 \\
\hline 1.0 & $2.5816 \mathrm{e}-5$ & 0.0062 \\
\hline 3.0 & 0.0010 & 0.0040 \\
\hline
\end{tabular}

Table 2: Greatest error obtained by applying SFD.

\begin{tabular}{|c|c|c|}
\cline { 2 - 3 } \multicolumn{1}{c|}{} & \multicolumn{2}{c|}{ Greatest error } \\
\hline$C$ & Lower branch & Upper branch \\
\hline 0.1 & $1.9310 \mathrm{e}-6$ & 0.0182 \\
\hline 1.0 & $3.4198 \mathrm{e}-5$ & 0.0058 \\
\hline 3.0 & $5.2045 \mathrm{e}-4$ & 0.0030 \\
\hline
\end{tabular}

Table 3: Greatest error obtained by applying NSFD. 
The results exhibit good precision for all values of $C$, with lower accuracy for the upper branch. It is also interesting to observe the different behavior that both lower and upper branch have in regard to the absolute error vector (the vector that contains the difference between the exact and computed solution for each value of $x$ ). In Figures 1 and 2 we can see that while in the case of the lower branch, the greatest error is located in the middle $(x=1 / 2)$, in the case of the upper branch there are two maximums located on either sides of it. Both figures have been obtained by applying $H G(\gamma)$, with $\gamma=1 / 2, C=1, n=20$ and a tolerance of $10^{-10}$.

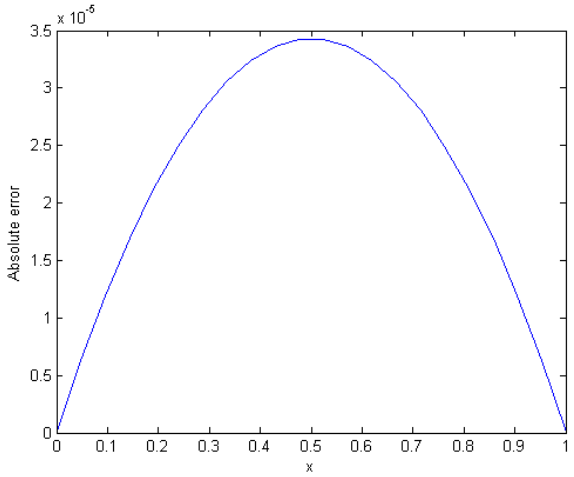

Fig. 1: Absolute lower branch error. $\mathrm{HG}(1 / 2), C=1$ and $n=20$.

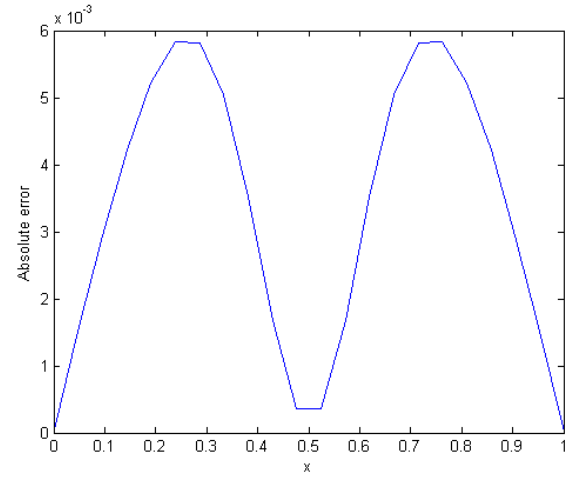

Fig. 2: Absolute upper branch error. $\mathrm{HG}(1 / 2), C=1$ and $n=20$.

Although there is almost no difference between the computed solution of the three studied methods, there is difference between the number of iterations that it takes for each method to reach the estimated solution. In Tables from 4 to 7 , it is shown, for the four mentioned members of the family $H G(\gamma)$ that minimize the asymptotical error, and for different values of $C$ and lower/upper branch (LB and UB, respectively), the number of iterations needed for our method $H G(\gamma)$ compared to the classical Newton' and Traub's schemes. Let us notice that all four tested members of the family not only converge always faster than Newton's method but also that Traub's scheme, in some cases. Regarding the number of iterations needed, there is no difference between using SFD and NSFD, since NSFD increases the accuracy of the discretization, but does not affect that of the iterative method.

\begin{tabular}{|c|c|c|c|c|c|c|}
\cline { 2 - 7 } \multicolumn{1}{c|}{} & \multicolumn{6}{|c|}{ No. of iter. $\gamma=1 / 2$} \\
\hline \multirow{2}{*}{$\mathrm{C}$} & \multicolumn{3}{|c|}{$\mathrm{LB}$} & \multicolumn{3}{c|}{$\mathrm{UB}$} \\
\cline { 2 - 7 } & $\mathrm{N}$ & $\mathrm{T}$ & $\mathrm{HG}(1 / 2)$ & $\mathrm{N}$ & $\mathrm{T}$ & $\mathrm{HG}(1 / 2)$ \\
\hline 0.1 & 2 & 1 & 1 & 5 & 3 & 3 \\
\hline 1.0 & 3 & 2 & 2 & 8 & 5 & 4 \\
\hline 3.0 & 4 & 3 & 2 & 6 & 4 & 3 \\
\hline
\end{tabular}

Table 4: Number of iterations required per method, $H G(1 / 2)$

\begin{tabular}{|c|c|c|c|c|c|c|}
\cline { 2 - 7 } \multicolumn{1}{c|}{} & \multicolumn{4}{c|}{ No. of iter. $\gamma=(6-\sqrt{3}) / 6$} \\
\hline \multirow{2}{*}{$\mathrm{C}$} & \multicolumn{3}{|c|}{ LB } & \multicolumn{3}{c|}{ UB } \\
\cline { 2 - 7 } & $\mathrm{N}$ & $\mathrm{T}$ & HG & $\mathrm{N}$ & $\mathrm{T}$ & HG \\
\hline 0.1 & 2 & 1 & 1 & 5 & 3 & 3 \\
\hline 1 & 3 & 2 & 1 & 8 & 5 & 4 \\
\hline 3 & 4 & 3 & 2 & 6 & 4 & 4 \\
\hline
\end{tabular}

Table 6: Number of iterations required per method, $H G((6-\sqrt{3}) / 6)$

\begin{tabular}{|c|c|c|c|c|c|c|}
\cline { 2 - 7 } \multicolumn{1}{c|}{} & \multicolumn{6}{|c|}{ No. of iter. $\gamma=3 / 2$} \\
\hline \multirow{2}{*}{$\mathrm{C}$} & \multicolumn{3}{|c|}{$\mathrm{LB}$} & \multicolumn{3}{c|}{$\mathrm{UB}$} \\
\cline { 2 - 8 } & $\mathrm{N}$ & $\mathrm{T}$ & $\mathrm{HG}(3 / 2)$ & $\mathrm{N}$ & $\mathrm{T}$ & $\mathrm{HG}(3 / 2)$ \\
\hline 0.1 & 2 & 1 & 1 & 5 & 3 & 3 \\
\hline 1 & 3 & 2 & 2 & 8 & 5 & 5 \\
\hline 3 & 4 & 3 & 2 & 6 & 4 & 4 \\
\hline
\end{tabular}

Table 5: Number of iterations required per method, $H G(3 / 2)$

\begin{tabular}{|c|c|c|c|c|c|c|}
\cline { 2 - 7 } \multicolumn{1}{c|}{} & \multicolumn{4}{c|}{ No. of iter. $\gamma=(6+\sqrt{3}) / 6$} \\
\hline \multirow{2}{*}{$\mathrm{C}$} & \multicolumn{3}{|c|}{ LB } & \multicolumn{3}{c|}{ UB } \\
\cline { 2 - 7 } & $\mathrm{N}$ & $\mathrm{T}$ & HG & $\mathrm{N}$ & $\mathrm{T}$ & HG \\
\hline 0.1 & 2 & 1 & 1 & 5 & 3 & 3 \\
\hline 1 & 3 & 2 & 2 & 8 & 5 & 5 \\
\hline 3 & 4 & 3 & 2 & 6 & 4 & 4 \\
\hline
\end{tabular}

Table 7: Number of iterations required per method, $H G((6+\sqrt{3}) / 6)$ 


\section{Conclusions}

A new parametric family of third-order methods, for solving nonlinear equations, is presented. Some elements of the family, that reduce the asymptotical error, have been used for approximating the solution of the planar 1D Bratu problem, with good stability properties.

\section{References}

1. J.R. Sharma, H. Arora, On efficient weighted-Newton methods for solving systems of nonlinear equations. Appl. Math. Comput. 222 (2013) 497-506.

2. J. R. Sharma, R. K. Guna, R. Sharma, An efficient fourth order weighted-Newton method for systems of nonlinear equations, Numer. Algor. 62 (2013) 307-323.

3. F. Awawdeh, On new iterative method for solving systems of nonlinear equations, Numer. Algor. 54 (2010) 395-409.

4. V. Kanwar, S. Kumar, R. Behl, Several New Families of Jarratts Method for Solving Systems of Nonlinear Equations, Applications and Appl. Math. 8(2) (2013) 701-716.

5. C. Andreu, N. Cambil, A. Cordero, J.R. Torregrosa, Preliminary Orbit Determination of Artificial Satellites: A Vectorial Sixth-Order Approach, Abstr. Appl. Anal., 2013 (Article ID 960582).

6. M. F. Abad, A. Cordero, J. R. Torregrosa, Fourth-and fifth-order for solving nonlinear systems of equations: An application to the global positioning system, Abstr. Appl. Anal., 2013 (Article ID 586708).

7. M. Petković, B. Neta, L. Petković, J. Džunić, Multipoint Methods for Solving Nonlinear Equations, Academic Press, Amsterdam, 2013.

8. H.H.H. Homeier, On Newton-tyoe methods with cubic convergence, J. Comput. Appl. Math. 176 (2005) 425-432.

9. A. Cordero, J.L. Hueso, E. Martínez, J.R. Torregrosa, A modified Newton-Jarratt's composition, Numer. Algor. 55 (2010) 87-99.

10. G. Bratu, Sur les equation integrals non-lineaires, Bull. Math. Soc. France 42 (1914) 113-142.

11. I.M. Gelfand, Some problems in the theory of quasi-linear equations, Trans. Amer. Math. Soc. Ser. 2 (1963) $295-381$.

12. J. Jacobsen, K. Schmitt, The Liouville-Bratu-Gelfand problem for radial operators, J. Differential Equations 184 (2002) $283-298$.

13. R. Jalilian, Non-polynomial spline method for solving Bratu's problem, Comput. Phys. Comm. 181 (2010) 1868-1872.

14. Y.Q. Wan, Q. Guo, N. Pan, Thermo-electro-hydrodynamic model for electrospinning process, Int. J. Nonlinear Sci. Numer. Simul. 5 (2004) 5-8.

15. J.F. Traub, Iterative Methods for the Solution of Equations, Chelsea Publishing Company, New York, 1982.

16. R. Buckmire, Applications of Mickens finite differences to several related boundary value problems, in: R.E. Mickens (Ed.), Advances in the Applications of Nonstandard Finite Difference Schemes, World Scientific Publishing, Singapore, 2005, 47-87.

17. J.P. Boyd, One-point pseudospectral collocation for the one-dimensional Bratu equation, Appl. Math. Comput. 217 (2011) $5553-5565$.

18. A. Mohsen, A simple solution of the Bratu problem, Comput. Math. with Appl. 67 (2014) 26-33. 